\title{
Die Ammonium-Konzentration im Venenblut von Säuglingen und Kindern
}

\author{
Von N. Liappis, J. Brodehl und A. JäkEL
}

Aus der Universitäts-Kinderklinik Bonn (Direktor: Prof. Dr. H. Hungerland)

(Eingegangen am 21. Juli 1970)

\begin{abstract}
Die direkte kolorimetrische Bestimmung von Ammonium ${ }^{1}$ ) im Blut mit Hilfe der Indophenol-Reaktion ermöglicht durch ihre Einfachheit und Spezifität. die routinemäßige Durchführung im klinisch-chemischen Laboratorium. Die Untersuchung von 65 Säuglingen und Kindern bis zu 3 Jahren, bei denen für Leberschäden, Stoffwechselstörungen und endokrine Störungen klinisch und anamnestisch keine Anhaltspunkte bestanden, ergab als normale Ammonium-Konzentration ${ }^{2}$ ) im Blut 121,9 $\pm 24,7 \mu \mathrm{g} / 100 \mathrm{~m} / \mathrm{Blut}$ (Mittelwert aus 65 Doppelbestimmungen) mit Schwankungen zwischen 69 und $179 \mu \mathrm{g} / 100 \mathrm{~m} /$ Blut. Im Vergleich hierzu beträgt die Ammonium-Konzentration im Blut bei Kindern zwischen 3 und 14 Jahren 104,3 $\pm 18,24 \mu \mathrm{g} / 100 \mathrm{~m} /$ Blut (Mittelwert aus 98 Doppelbestimmungen) mit Schwankungen zwischen 64 und $152 \mu \mathrm{g} / 100 \mathrm{~m} /$ Blut. Bei Lebererkrankungen und Störungen im Harnstoffzyklus wurden höhere Ammonium-Konzentrationen im Blut gemessen.
\end{abstract}

\section{The ammonium concentration in the venous blood of sucklings and cbildren}

The direct colorimetric determination of ammonium in blood with the aid of the indophenol reaction is simple and specific and suitable for routine measurements in the clinical chemical laboratory. The study of 65 sucklings and children up to age 3 years, in which there was no clinical or anamnestic evidence of liver damage, metabolic or endocrine disorders, gave normal values ${ }^{2}$ of $121,9 \pm 24,7 \mu \mathrm{g}$ ammonium $/ 100 \mathrm{~m} l$ blood (average of 65 duplicates) with a scatter of $69-179 \mu \mathrm{g} / 100 \mathrm{~m} / \mathrm{blood}$. By comparison, the blood of $3-14$ year olds contained $104,3 \pm 18,24 \mu \mathrm{g} / 100 \mathrm{~m} l$ blood (average of 98 duplicates) with a scatter of $64-152 \mu \mathrm{g} / 100 \mathrm{~m} l$ blood. Elevated concentrations of blood ammonium were found in liver disease and disorders of the urea cycle.

Die bisher beschriebenen Verfahren der Ammoniumbestimmung im Blut können in zwei Gruppen zusammengefaßt werden. In der ersten Gruppe wird Ammonium zunächst nach einer der folgenden $\mathrm{Me}-$ thoden möglichst spezifisch und quantitativ isoliert durch:

a) Destillation (5)

b) Aeration $(6,7)$

c) Diffusion bzw. Mikrodiffusion (8-30)

d) Adsorption an einen Ionenaustauscher (31-38).

Anschließend wird Ammonium selbst titrimetrisch oder photometrisch (nach Farbreaktion mit NESSLER's Reagenz, reduziertem Ninhydrin, Phenol-Hypochlorit-Reagenz oder Hypobromit-Phenosaphranin-Reagenz) bestimmt. In der zweiten Gruppe wird die AmmoniumKonzentration direkt im Gewebs- oder Blutextrakt nach Enteiweißung gemessen. Nach der Enteiweißung mit Trichloressigsäure, Perchlorsäure oder Natriumwolframat-Schwefelsäure erfolgt die Bestimmung mit Phenol-Hypochlorit-Reagenz (39-45, 51) oder auf enzymatischem Wege (44, 46-48).

In der vorliegenden Arbeit wurde zur Aufstellung von Normalwerten für die Ammonium-Konzentration im Blut von Säuglingen und Kindern das von MACCuLLOUGH (43) angegebene Bestimmungsverfahren angewandt. Es basiert auf der BERTHELOT'schen Reaktion (49) des Ammoniaks mit Indophenol (Reaktions-

1) Der pK-Wert des Dissoziationsgleichgewichtes $\mathrm{NH}_{4}^{+} \rightleftharpoons \mathrm{NH}_{3}$ $+\mathrm{H}^{+}$beträgt 9,2. Im Blut und im Gewebe sind fast nur Ammoniumionen vorhanden. Als Ammonium wird die Summe des dissoziierten und undissoziierten Anteils bezeichnet. Die Summe wird bei allen Bestimmungsmethoden ermittelt.

$\left.{ }^{2}\right)$ Alle Angaben als $\mathrm{NH}_{4}^{+}-\mathrm{N}$. mechanismus bei l. c. (50)). Der gebildete Farbstoff ist in alkalischer Lösung stabil. HoRN und SQuire (34) fanden, daß die Empfindlichkeit der Reaktion durch Zugabe von Hypochlorit zu der alkalischen Natriumphenolat-Lösung erhöht wird. Nach SEARCY und Mitarbeitern (51) ist aber die Reaktion bei umgekehrter Reihenfolge der zugegebenen Reagenzien spezifischer.

\section{Material und Methoden}

\section{Reagenzien}

1. Lösung I: $10 \mathrm{~g}$ Phenol (Fa. Merck 206) p. a. und $50 \mathrm{mg} \mathrm{Na}$ triumpentacyanonitrosylferrat-[II] (Fa. Merck 6541) p. a. ad $1 \mathrm{l}$ Wasser.

2. Lösung II: $5 \mathrm{~g}$ Natriumhydroxid (Fa. Mcrck 6495) p. a., $53,7 \mathrm{~g}$ Dinatriumbydrogenphosphat $\left(\mathrm{Na}_{2} \mathrm{HPO}_{4} \cdot 12 \mathrm{H}_{2} \mathrm{O}\right)$ (Fa. Merck 6579) p. a. und $10 \mathrm{ml}$ Natriumbypochlorit-Lösung ( $\mathrm{Fa}_{\text {a }}$ Bayer AO 106) techn. (12\% Mindestgehalt an aktivem Chlor, Angabe der $\mathrm{Fa}$. Bayer) ad $1 l$ Wasser.

3. 10proz. Natriumwolframat-Lösung ( $\mathrm{Fa}$. Merck 6673 ) p. a.

4. $1 \mathrm{~N}$ Schwefelsäure (Fa. Merck 9981) Titrisol.

5. Standardlösung: $0,471 \mathrm{~g}$ Ammoniumsulfat (Fa. Merck 1217) p. a. ad $1 l$ Wasser (entsprechend $100 \mu \mathrm{g} \mathrm{NH}+-\mathrm{N} / \mathrm{m} l$ ).

Lösung I und II sollen bei $4^{\circ}$ aufbewahrt werden und besitzen wie die Standardlösung eine Haltbarkeit von einem Monat.

\section{Versuche}

Die Bestimmung det Ammonium-Konzentration im Blut wurde bei insgesamt 28 Säuglingen und 135 Kindern durchgeführt; die Altersverteilung ist in Tabelle 1 dargestcllt. Ebenso wurde Ammonium bei 12 kranken Kindern bestimmt, deren Diagnose in der Tabclle 2 aufgefühtt wird.

\section{Ammonium-Bestimmung}

Venöses Blut wird möglichst ungestaut $\mathrm{dem}$ nüchternen ruhenden Patienten entnommen und in einem mit 2-3 Tropfen Heparin (Liquemin) versetzten Zentrifugenröhrchen aufgefangen. $2 \mathrm{~m} l$ 
Tab. 1

Aufteilung der 163 untersuchten Säuglinge und Kinder nach dem Alter

\begin{tabular}{|c|c|c|c|c|c|c|c|c|c|c|}
\hline & $\begin{array}{c}\text { Bis } 3 \mathrm{Mo} \\
15\end{array}$ & $3 \mathrm{Mo}_{13}-1 \mathrm{~J}$ & $1 \mathrm{~J}-2 \mathrm{~J}$ & $\frac{2 \mathrm{~J}-3 \mathrm{~J}}{20}$ & $3 \mathrm{~J}-4 \mathrm{~J}$ & $4 \mathrm{~J}-5 \mathrm{~J}$ & $5 \underset{12}{5-6} \mathrm{~J}$ & $6 \underset{4}{-7} \mathrm{~J}$ & $7 \underset{10}{\mathrm{~J}-8} \mathrm{~J}$ & $8 \mathrm{~J}-14 \mathrm{~J}$ \\
\hline
\end{tabular}

Tab. 2

Aufteilung der 12 untersuchten Kinder nach Art der Erkrankung und Blut-Ammonium-Konzentration

\begin{tabular}{|c|c|c|c|}
\hline Nr. & Tag & Diagnose & $\mathrm{NH}_{4}^{+}-\mathrm{N}(\mu \mathrm{g} / 100 \mathrm{ml})$ \\
\hline 1 & $\begin{array}{r}1 \\
10\end{array}$ & Mangelgeburt, Dystrophie & $\begin{array}{l}200 \\
145\end{array}$ \\
\hline 2 & $\begin{array}{r}1 \\
42 \\
81\end{array}$ & Periodische Ammoniumintoxikation durch Ornithin-Transcarbamylase-Defekt & $\begin{array}{l}130 \\
195 \\
495\end{array}$ \\
\hline 3 & $\begin{array}{r}1 \\
84\end{array}$ & Leberstauung bei Pleuramesoendotheliom & $\begin{array}{l}176 \\
139\end{array}$ \\
\hline 4 & 1 & Neugeborenen-Hyperbilirubinämie & 119 \\
\hline 5 & 1 & Transfusions-Hepatitis & 172 \\
\hline 6 & 1 & Zustand nach Hepatitis & 110 \\
\hline 7 & $\begin{array}{r}1 \\
14 \\
17\end{array}$ & Congenitale Gallengangatresie & $\begin{array}{l}110 \\
204 \\
318\end{array}$ \\
\hline 8 & 1 & Urämie bei beidseitiger Hydronephrose & . 172 \\
\hline 9 & $\begin{array}{r}1 \\
20\end{array}$ & Neonatale Hepatitis & $\begin{array}{l}166 \\
126\end{array}$ \\
\hline 10 & 1 & Coeliakie & 130 \\
\hline 11 & 1 & Akute Lymphoblasten-Leukämie & 105 \\
\hline 12 & 1 & Akute Lymphoblasten-Leukämie & 115 \\
\hline
\end{tabular}

Blut werden sofort in $1 \mathrm{ml} 10$ proz. Natriumwolframat-Lösung pipettiert und $1 \mathrm{ml} 1 \mathrm{~N}$ Schwefelsäure zugegeben. Danach wird gut gemischt und $10 \mathrm{Min}$. bei $3000 \mathrm{U} / \mathrm{Min}$. zentrifugiert. $\mathrm{Zu} 0,5 \mathrm{ml}$ des klaren, proteinfreien Überstandes werden je $2,5 \mathrm{ml}$ der Lösung I und II pipettiert und nach jeder Zugabe gut gemischt. Anschließend wird für $35 \mathrm{Min}$. bei $37^{\circ}$ inkubiert und die Lichtabsorption des entstandenen Farbstoffs bei $625 \mathrm{~nm}$ und einer Schichtdicke von $1 \mathrm{~cm}$ gemessen.

Zur Kontrolle werden bei jeder Meßreihe ein Reagenzienleerwert und 5 Standardwerte $(25,50,75,100$ und $150 \mu \mathrm{g} \mathrm{NH}+-\mathrm{N} / 100 \mathrm{ml})$ mitbestimmt.

Die Berecbnung der Ammonium-Konzentration im Blut erfolgt nach folgender Formel:

$$
\frac{E_{\text {Probe }}-E_{\text {Leerwert }}}{\text { EStandard }_{\text {Seerwert }}} \cdot \mu \mathrm{g} / 100 \mathrm{~m} l \text { Standard }=
$$

Als Standard wird jeweils der Wert zur Berechnung genommen, der der Konzentration des Analysenwertes am nächsten steht. Mit Hilfe der Standardwerte kann auch eine Eichkurve angelegt werden, aus der die gemessenen Extinktionswerte der einzelnen Analysen in $\mu \mathrm{g} \mathrm{NH}_{4}^{+}-\mathrm{N} / 100 \mathrm{~m} /$ Blut berechnet werden können (s. Abb. 1).

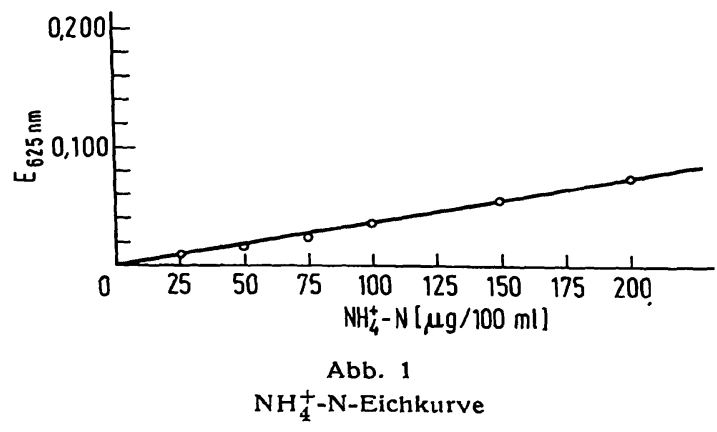

Spezifität der Methode

Nach Okuda und Mitarbeitern (52) inhibiert die Zugabe von Natriumwolframat-Lösung und Schwefelsäure die Farbreaktion der Nichtprotein-Stickstoff-Verbindungen mit Indophenol mit Ausnahme von Ammonium für eine Inkubationszeit von $60 \mathrm{Min}$. bei $37^{\circ}$. Die Ammonium-Standardlösungen zeigen nach $35 \mathrm{Min}$. Inkubationszeit das Maximum ihrer Farbintensität und OKuDA und Mitarbeiter fanden, daß die Verbindungen Leucin, Hąrnsäure,
Harnstoff, Kreatinin, Adrenalin, Noradrenalin, Histamin und Acetylcholin auch in hohen Konzentrationen nach $35 \mathrm{Min}$. Inkubationszeit die Farbentwicklung in keiner Weise beeinflussen. Sie geben an, daß Prolin in sechsfacher Normalkonzentration den Ammoniumwert um 20\% erniedrigt.

Wiederfindung von Ammonium aus dem Blut

Zur Bestimmung der Wiederfindung von Ammonium aus dem Blut wurde $1 \mathrm{~m} l$ Blut zu $1 \mathrm{~m} l$ Natriumwolframat-Iösung gegeben. Hierzu wurde jeweils $1 \mathrm{ml}$ der 25, 50, 75, 100, 150 und $200 \mu \mathrm{g} \mathrm{NH}{ }_{4}^{+}-\mathrm{N} / 100 \mathrm{~m} l$ Standardlösung pipettiert und mit $1 \mathrm{ml} 1 \mathrm{~N}$ Schwefelsäure versetzt. Nach Mischen und Zentrifugieren wurden vom Úberstand $0,5 \mathrm{ml}$ zur Bestimmung der Ammonium-Konzentration verwandt. Zur Ermittlung der Wiederfindung wurde der Blut-Leerwert von den erhaltenen Werten subtrahiert. Die Ergebnisse sind in Tabelle 3 zusammengestellt.

Tab. 3

Wiederfindung von Ammonium aus dem Blut

\begin{tabular}{ccc}
\hline $\begin{array}{c}\text { Zugesetzte Menge an } \\
\mathrm{NH}_{\mathbf{4}}^{+-N}\end{array}$ & $\begin{array}{c}\text { Wiedergefundene } \\
\text { Menge an } \mathrm{NH}_{\mathbf{4}}^{+}-\mathrm{N}\end{array}$ & Wiederfindung \\
\hline$(\mu \mathrm{g} / 100 \mathrm{~m} l)$ & $(\mu \mathrm{g} / 100 \mathrm{~m} l)$ & $(\%)$ \\
\hline 25 & 26,24 & 104,96 \\
50 & 45,48 & 90,96 \\
75 & 72,76 & $96,101,3$ \\
100 & 105,107 & 105,107 \\
150 & 138,147 & 92,98 \\
200 & 186,195 & $93,97,5$ \\
\hline
\end{tabular}

\section{Ergebnisse und Diskussion}

In Tabelle 4 ist die Streubreite, der Mittelwert, die Standardabweichung und der Variationskoeffizient der Ammonium-Konzentration im venösen Blut verschiedener Altersgruppen dargestellt.

Wie aus Tabelle 4 hervorgeht, kann man die Ammonium-Konzentrationen im Blut in den verschiedenen Altersgruppen in zwei Hauptgruppen zusammenfassen. Die erste Hauptgruppe schließt die Werte bis zu 3 Jahren (s. Abb. 2) und die zweite die Werte von 3 Jahren bis zu 14 Jahren ein (s. Abb. 3). 
Tab. 4

Ammonium-Konzentration im venösen Blut bei Säuglingen und Kindern verschiedenen Alters

\begin{tabular}{|c|c|c|c|c|}
\hline Altersgruppen & $\begin{array}{c}\text { Streubreite } \\
\mathrm{NH}_{4}^{+}-\mathrm{N} \\
(\mu \mathrm{g} / 100 \mathrm{~m} l \text { Blut })\end{array}$ & $\begin{array}{c}\text { Mittelwert } \\
\mathrm{NH}_{4}^{+}-\mathrm{N} \\
(\mu \mathrm{g} / 100 \mathrm{ml} \text { Blut })\end{array}$ & $\begin{array}{c}\text { Standardab- } \\
\text { weichung } \\
\mathrm{NH}_{4}^{+-\mathrm{N}} \\
(\mu \mathrm{g} / 100 \mathrm{~m} l \text { Blut) }\end{array}$ & $\begin{array}{c}\text { Variationskoeff. } \\
\qquad \%\end{array}$ \\
\hline $\begin{array}{l}\text { Bis } 3 \text { Monate } \\
3 \mathrm{Mon}-1 \mathrm{~J} \\
1 \mathrm{~J}-2 \mathrm{~J} \\
2 \mathrm{~J}-3 \mathrm{~J} \\
3 \mathrm{~J}-4 \mathrm{~J} \\
4 \mathrm{~J}-5 \mathrm{~J} \\
5 \mathrm{~J}-6 \mathrm{~J} \\
6 \mathrm{~J}-7 \mathrm{~J} \\
7 \mathrm{~J}-8 \mathrm{~J} \\
8 \mathrm{~J}=14 \mathrm{~J} \\
\text { Erwachsene }\end{array}$ & $\begin{array}{r}72-179 \\
100-177 \\
96-174 \\
69-162 \\
85-135 \\
78-152 \\
67-124 \\
71-122 \\
72-120 \\
64-146 \\
40-109\end{array}$ & $\begin{array}{r}123,1 \\
125,9 \\
120,5 \\
118,2 \\
108,7 \\
110,9 \\
97,3 \\
98,3 \\
108,2 \\
102,3 \\
78,9\end{array}$ & $\begin{array}{l}29,29 \\
24,84 \\
22,45 \\
22,23 \\
15,10 \\
21,66 \\
15,30 \\
22,18 \\
14,59 \\
20,60 \\
20,59\end{array}$ & $\begin{array}{l}23,8 \\
19,7 \\
18,7 \\
18,8 \\
13,9 \\
19,5 \\
15,8 \\
22,6 \\
13,5 \\
20,2 \\
26,0\end{array}$ \\
\hline
\end{tabular}

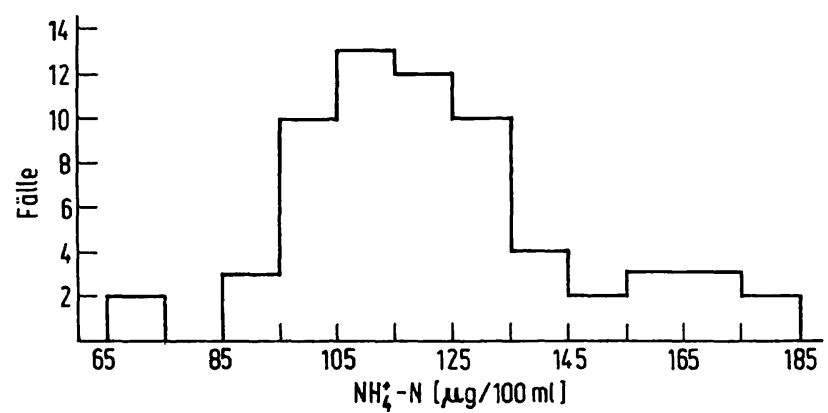

Abb. 2

Die Verteilung der Blutkonzentration von $\mathrm{NH}_{4}^{+}-\mathrm{N}$ bei 65 Säuglingen und Kindern im Alter bis zu drei Jahren

Es läßt sich hieraus ein Ammoniumgehalt ${ }^{2}$ ) des venösen Bluts von Säuglingen und Kindern bis zu 3 Jahren von $121,9 \mu \mathrm{g} / 100 \mathrm{ml}$ Blut mit Schwankungen zwischen 69 und $179 \mu \mathrm{g} / 100 \mathrm{ml}$ Blut und von 3 Jahren bis $\mathrm{zu} 14$ Jahren von $104,3 \mu \mathrm{g} / 100 \mathrm{ml}$ Blut mit Schwankungen zwischen 64 und $152 \mu \mathrm{g} / 100 \mathrm{ml}$ Blut berechnen. Die Standardabweichung beträgt bei der ersten Gruppe $24,7 \mu \mathrm{g} / 100 \mathrm{ml}$ Blut und bei der zweiten $18,24 \mu \mathrm{g} /$ $100 \mathrm{ml}$ Blut.

Im Vergleich $z u$ diesen mit der direkten, kolorimetrischen Methode ermittelten Werten, bestimmten SeCCHI und Mitarbeiter (35) mit Hilfe des Diffusionsverfahrens bei ihren Untersuchungen einen Extrem- bereich bei Säuglingen (0-14 Tage) von $90-150 \mu \mathrm{g} /$ $100 \mathrm{ml}$ Blut und bei Kindern von $7-63 \mu \mathrm{g} / 100 \mathrm{ml}$ Blut. Die bei 12 Kindern mit Leberstörungen gemessenen Werte sind in Tabelle 2 dargestellt.

Mit Hilfe der direkten, kolorimetrischen Methode wurde auch Ammonium im Blut von 22 Erwachsenen bestimmt. Der aus diesen Untersuchungen (Doppelbestimmungen) errechnete Mittelwert der Normalkonzentration von $78,9 \pm 20,6 \mu \mathrm{g} \mathrm{NH}_{4}^{+}-\mathrm{N} / 100 \mathrm{ml}$ Blut

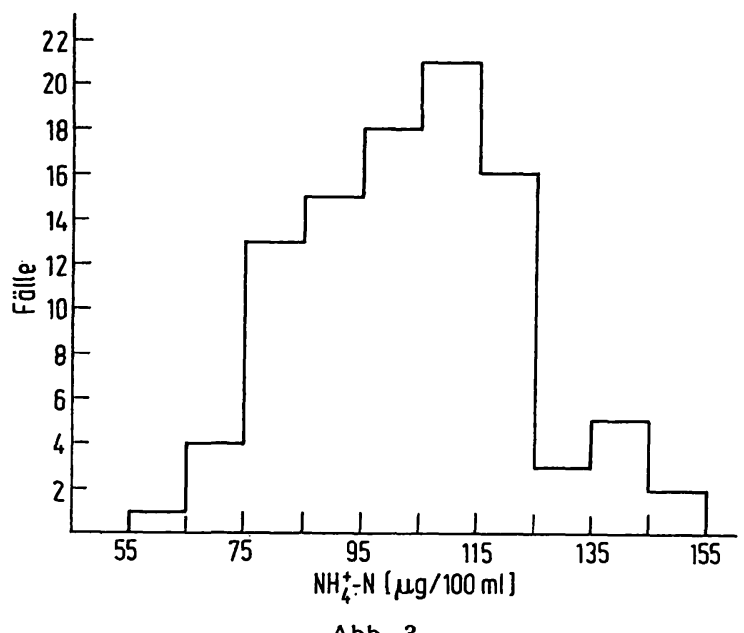

Abb. 3

Die Verteilung der Blutkonzentration von $\mathrm{NH}_{4}^{+}-\mathrm{N}$ bei 98 Kindern im Alter bis zu 14 Jahren
Abb. 4

Zusammenstellung der in der Literatur angegebenen Werte für die normale Ammonium-Konzentration des Blutes Erwachsener

Sgl = Säuglinge $\mathrm{Kdr}=\mathrm{Kinder}$

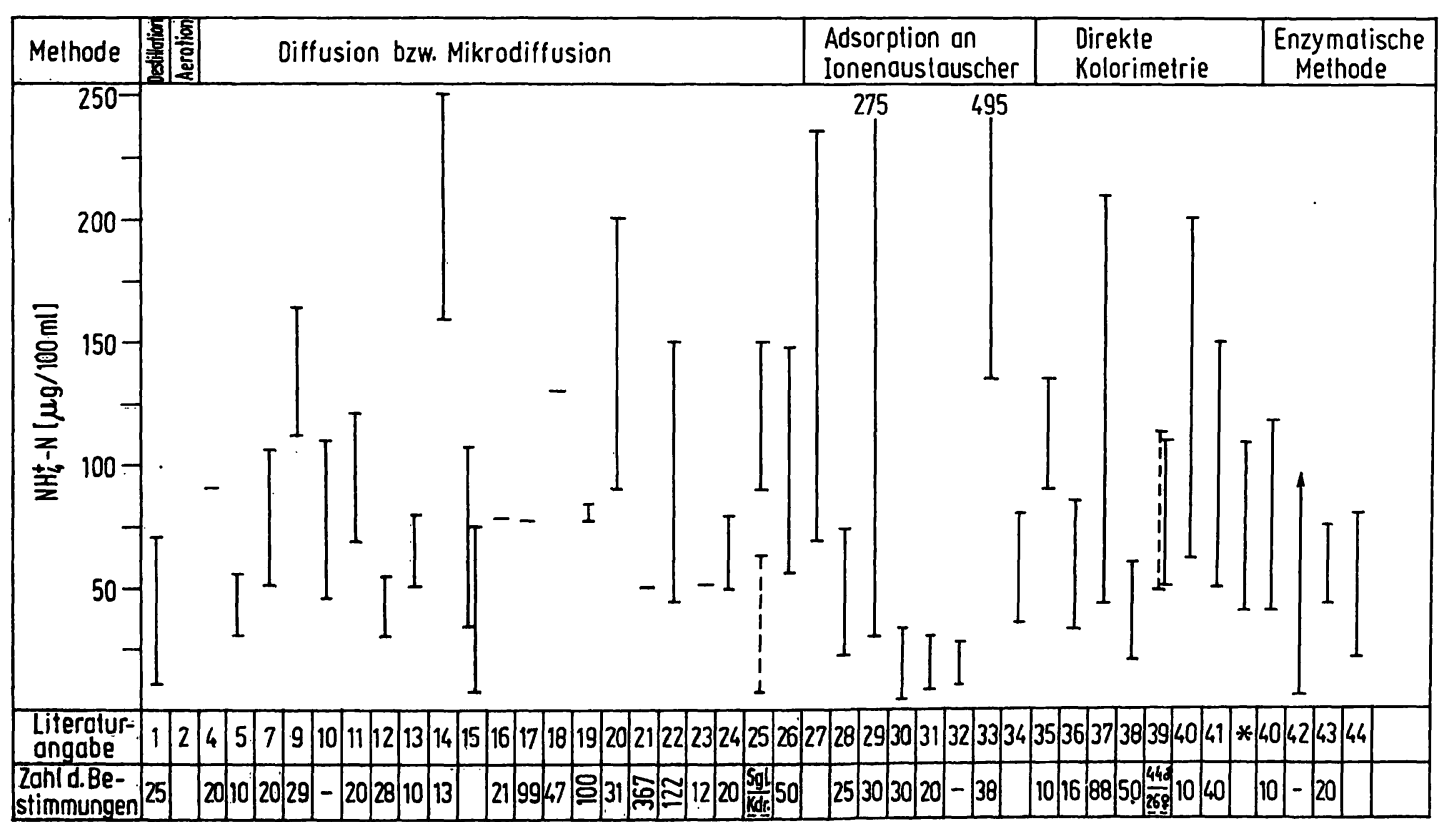


stimmt weitgehend mit denen der Literatur überein (39-45, 51). Der gemessene Extrembereich betrug $40-109 \mu \mathrm{g} \mathrm{NH} \mathrm{NH}_{4}^{+} \mathrm{N} / 100 \mathrm{ml}$ Blut und der Variationskoeffizient $26 \%$.

Aus der Fülle der verschiedenen Bestimmungsverfahren sowie aus der Unterschiedlichkeit der ermittelten Normalwerte im venösen Blut von Erwachsenen ist ersichtlich, daß die Bestimmung der im $\mu \mathrm{g} / 100 \mathrm{ml}$-Bereich befindlichen Ammonium-Konzentration mit erheblichen Schwierigkeiten und Fehlerquellen verbunden sein kann.

Die in der vorliegenden Arbeit angewandte direkte kolorimetrische Methode eignet sich wie auch die übrigen direkten kolorimetrischen Verfahren besonders für Untersuchungen im klinisch-chemischen RoutineLaboratorium. Sie ist wesentlich einfacher und weniger zeitraubend, insbesondere im Vergleich zu den Diffusionsverfahren. Hinzu kommt, daß Spezialapparate nicht benötigt werden und eine Kontaminierung, die eine zeitraubende Reinigung der Geräte beanspruchen würde, wie z.B. für die enzymatischen und DiffusionsBestimmungsmethoden, nicht auftreten kann.
Im Vergleich zu den anderen direkten kolorimetrischen Bestimmungsverfahren besitzt die von McCullough beschriebene Bestimmungsmethode den Vorteil, daß die Blutproben nicht sofort analysiert werden müssen. Dieses ist besonders bei Belastungstests mit Eiweiß oder Ammoniumchlorid von Bedeutung.

McCullough fand, daß die enteiweißten Blutproben bei Raumtemperatur $1 \mathrm{Std}$., bei. $4^{\circ} 24 \mathrm{Stdn}$. und nach Abnahme des Überstandes bei Raumtemperatur 90 Min. und bei $-4^{\circ} 72$ Stdn. aufbewahrt werden können, ohne einen Einfluß auf die Ammonium-Konzentration zu verzeichnen.

Dank der oben beschriebenen Eigenschaften der direkten kolorimetrischen Bestimmung der AmmoniumKonzentration im Blut nach McCullough stellt sie eine für das klinisch-chemische Routine-Laboratorium geeignete Bestimmungsmethode dar.

Fräulein H. BARTH danken wir für die Durchführung dex Bestimmungen.

Die vorliegenden Untersuchungen wurden mit Unterstützung der Deutschen Forschungsgemeinschaft durchgeführt.

\section{Literatur}

1. Hennrich, G. und H. Breuer, Dtsch. med. Wschr. 84, 1415 (1959). - 2. LeVin, B., V. G. Oberholzer und I. Sinclair, Lancet London 1969 II, 170. - 3. KNAufF, H. G., D. Seybold und H. W. RAUB, Z. Gastroenterol. 3, 81 (1965). - 4. KNAUfF, H. G., H. HamelaranN, D. Seybold und A. Kanters, Klin. IVschr. 44, 147 (1966). - 5. Burgess, P. V. D. und H. W. MOORE, Clin. Chim. Acta Amsterdam 8, 162 (1963). - 6. SLYKE, B. D. vaN und A. Hrller, J. biol. Chemistry 102, 499 (1933). - 7. Stanojevic, L., Die physiologische und klinische Bedeutung des Blutammoniaks, Theodor Steinkopf, Dresden und Leipzig (1938). 8. Borsook, H., J. biol. Chemistry 110, 481 (1935). - 9. Brown, R. H., G. D. Duda, S. Korkes und P. Handler, Arch. Biochem. Biophysics 66, 301 (1957). - 10. Conway, E. J., Microdiffusion analysis and volumetric error, 4th revised edit., Crosby Lockwood \& Itd., London (1957). - 11. NathaN, D. G. und F. L. RodkEY, J. Laborat. clin. Med., S. Louis 49, 779 (1957). - 12. Russel, J. A., J. biol. Chemistry 156, 457 (1944). - 13. Seligson, D. und K. Kirahara, J. Laborat. clin. Med., S. Louis 49, 962 (1957). - 14. TAKatsukr, K., Sogo Igaku 16, 945 (1959), Zit. nach Chem. Abstr. 57, 16992b (1962). - 15. Ternberg, J. L. und F. B. Hershey, J. Laborat. clin. Med., S. Louis 56, 766 (1960). 16. WelleR, H., Röntgen- u. Lab.-Praxis 15, L 77, L 142 (1962). 17. MCDermott, W. V. und R. D. Adams, J. clin. Invest. 33, 1 (1954). - 18. Schwartz, R., G. B. Phillips, G. J. Gabuzda jr. und $C_{\text {H. }}$ S. Davidson, J. Laborat. clin. Med., S. Louis 42, 499 (1953). - 19. Hulme, W. A. und A. C. Cooper, J. Med. Laborat. Techn. 543 (1956). - 20. Bessman, S. P. und A. N. Bessman, J. clin. Invest. 34, 662 (1955). - 21. Phear, E. A., S. Sherlock und W: H. J. Summerskill, Lancet London 1955 I, 836. 22. SeEgmüller, J. E., R. Schwarz und C. S. Davidson, J. clin. Invest. 33, 984 (1954). - 23. Calkins, W. G., J. Laborat. clin. Med., S. Louis 47, 343 (1956). - 24. Erseman, B., W. Bakeweli und G. Cllark, Amer. J. Med. 20, 890 (1956). - 25. VAraY, A. und M. Mason, Gastroenterology Baltimore 86, 421 (1956). 26. Zimmerman, H. J. und R. J. Korn, Amer. J. med. Sci. 231,
177 (1956). - 27. Gangolli, S. und T. F. Nicholson, Clin Chim. Acta, Amsterdam 14, 585 (1966). - 28. Bưys Ballot A. F. K. und C. Steendijk, Clin. Chim. Acta, Amsterdam 12, 55 (1965). - 29. O'Brien und IBbotr, Laboratory Manual of Pediatric Micro- and Ultramicro-Biochemical Techniques, 3. Aufl, S. 31, 1962. Hoeber, Haxper \& Row, New York. - 30. ConN et al., Stand. Meth. clin. Chem. 5, 43 (1965). - 31. Foreman, J. A., Clin. Chem., N. Y. 10, 497 (1964). - 32. Hutchinson, J. H. und D. H. LabBY, J. Laborat. clin. Med., S. Louis 60, 170 (1962). 33. Fenton, J. C. B., Clin. Chim. Acta, Amsterdam 7, 163 (1962). 34. Horn, D. B. und Ck. R. Squrre, Clin. Chim. Acta, Amsterdam 14, 185 (1966). - 35. Secchr et al., Clin. Chim. Acta, Amsterdam 12, 235 (1965). - 36. Miller, G. E. und J. D. RiCE, Amer. J. clin. Path. 39, 97 (1963). - 37. Acland, J. D., Lancet London 1965 II, 345. - 38. Dienst, S. G. und B. Morris, J. Laborat. clin. Med., S. Louis 64, 495 (1964). - 39. KonItzER, K. und S. Vorgt, Clin. Chim. Acta Amsterdam 8, 5 (1963). - 40. MüllerBeissenhirtz, W. und H. Keller, Klin. Wschr. 43, 43 (1965). 41. Keller, H., W. Müller-Beissenfirtz und E. NeumanN, Klin. Wschr. 45, 314 (1967). - 42. WAGNER, K., G. SCHNEIDER und D. Theuer, Dtsch. Gesd-Wesen 23, 2459 (1968). - 43. McCuliovgh, H., Clin. Chim. Acta Amsterdam 17, 297 (1967). 44. GeroK, W. und J. PausCh, Z. ges. exp. Med. 148, 337 (1968). 45. McCullougri, H., Clin. Chim. Acta, Amsterdam 19, 101 (1968). - 46. Kirsten, E., C. Gerez und R. Kirsten, Biochem. Z. 337, 312 (1963). - 47. Oreskes, I., C. HirsCh und S. Kupfer, Clin. Chim. Acta, Amsterdam 26, 185 (1969). - 48. Mondzak, A., G. E. Ehritch und J. E. Seegmiller, J. Laborat. clin. Med., S. Louis 66, 526 (1965). - 49. Berthelot, M., Report Chim. Appl. 1, 281 (1859). - 50. SlyKe, B. D. vaN und A. HrLLER, J. biol. Chemistry 102, 499 (1933). - 51. SEARCY, R. L., N. M. Simms, J. A. Foreman und L. M. Begquist, Clin. Chim. Acta, Amsterdam 12, 170 (1965). - 52. OKuDA, H., S. Fujrr und Y. Kawashima, Tokushima J. Exptl. Med. 12, 11 (1965).

Dr. rer. nat. N. Liappis

Klin.-chem. Abteilung der UniversitätsKinderklinik

53 Bonn

Adenauerallee 119 
Wir bieten an:

\section{Serumproteine}

Anti-Gc / Gm / InV und Kontrollseren

Plasmaprotein - Antiseren

Australia: Antigen und Antikörper

Rheuma - Latex - Schnelltest
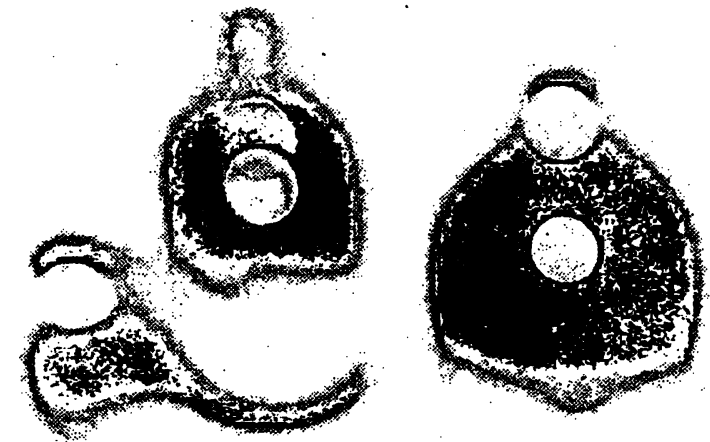

Pallues
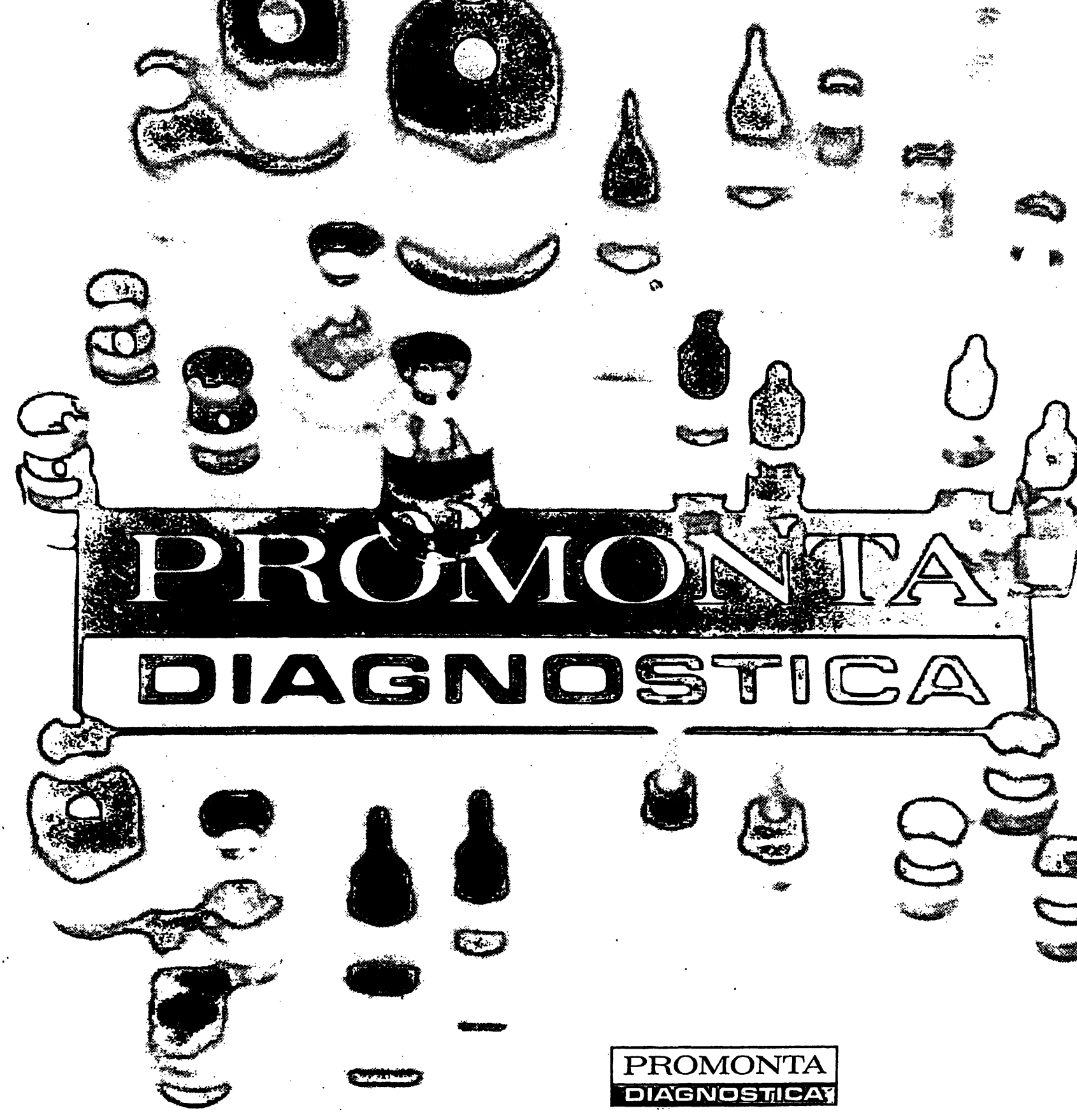

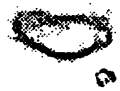
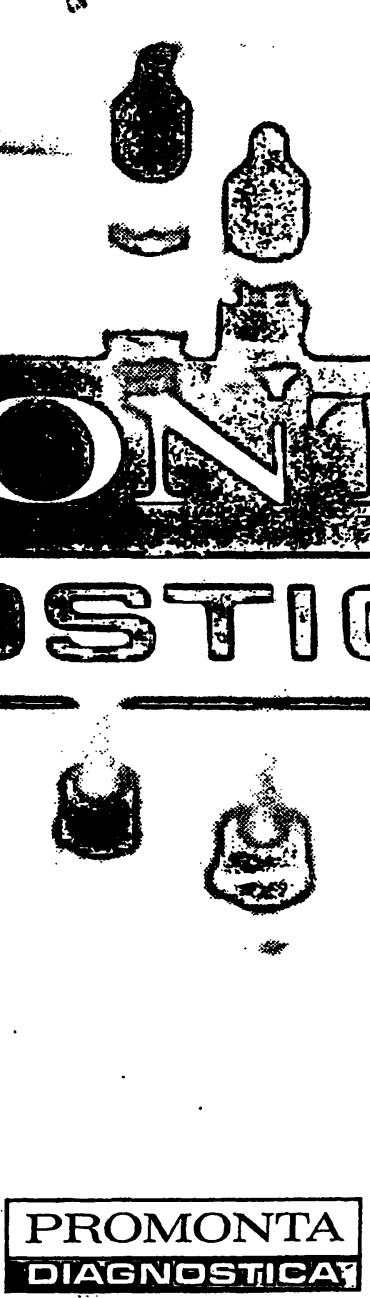

2000 Hamburg $70 \cdot$ Postfach 700148

Telefon (0411) $6936071-2 \cdot$ Telex 2174253 


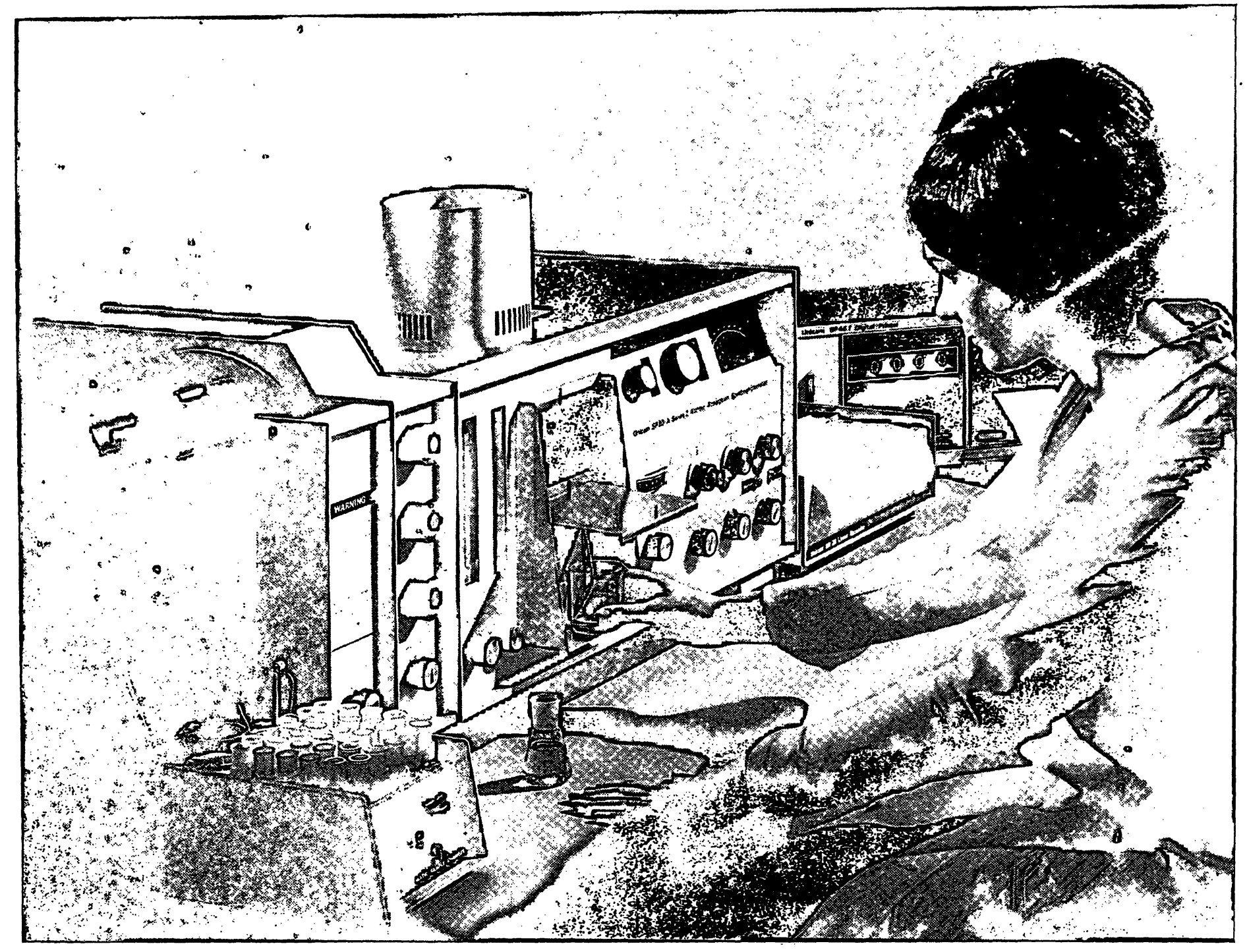

\title{
Seiem Sie anspruchswoller bei lhren Forschungs- und Routinearbeiten
}

\author{
nützen Sie die neuesten Möglichkeiten aus dem Programm PYE UNICAM
}

Das weiterentwickelte AtomabsorptionsSpektralphotometer SP 90 Serie 2 basiert auf dem Prinzip des bekannten SP 90. Es bietet Ihnen neue Vorteile durch seine lineare Extinktions-Anzeige, die kontinuierliche Skalendehnung, eine neue Lachgasbrenner-Einheit und die eingebaute Flammen-Emissions-Einheit. Zahlreiches Zubehör erweitert die Möglichkeiten: ein automatischer Probenwechsler, eine digitale Anzeige- und Drucker-Einheit, ein Lampenwechsler und ein Mehrschlitzbrenner.
Wellenlängenbereich $190 \mathrm{~nm}$ bis $770 \mathrm{~nm}$ bzw. $190 \mathrm{~nm}$ bis $852 \mathrm{~nm}$; Anschluß für Schreiber.

Anwendung: Agri-Kultur-Chemie, Biochemie, klinische Chemie, Galvanik, Nahrungsmittel, Baumaterialien, allgemeine Industrie-Chemie, Geologie, Metallurgie, Petro-Chemie, Kunststoffe, Wasser-Analysen, Textilien. Damit erschließt die Atomabsorptions-Technik der Analytik neue Möglichkeiten. In den Anwendungslaboratorien in Hamburg und Cambridge werden ständig neue Methoden ausgearbeitet.
Bitte fordern Sie ausführliches Informationsmaterial an. Es liegt für Sie bereit.

Philips Elektronik Industrie $\mathrm{GmbH}$ 2000 Hamburg $63 \cdot$ Röntgenstraße 22 Telefon (0411) 501031

Telefon-Nummern der Büros in: Berlin (0311) 245908 , Bielefeld (0521) 230 81. Dortmund (0231) 4 1961, Oüsseldorf (0211) 346051 , Frankfurt (0611) 79131 , Hamburg (0411) 28 92-1, Hannover (0511)
16601 . Kiel (0431) 732386 . Köln (0221) 514260 , Mannheim (0621) 42016 . München (0811) 76791 , Nürnberg (0911) 464763 , Stuttgar (0711) $589081-83$.

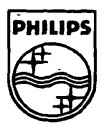

\section{Analysengeräte}

Wir interessieren uns für das Spektralphotometer SP 90 Serie 2 und bitten um

$\square$ Zusendung ausführlicher Unterlagen ein Angebot

Besuch Ihres Beratungsingenieurs

Gewünschtes bitte ankreuzen oder ergänzen 